\title{
Period analysis for simultaneous multichannel photometric observations
}

\author{
J. You ${ }^{1}$, J. Pelt ${ }^{1,2}$, and I. Tuominen ${ }^{1}$ \\ 1 Astronomy Division, Department of Physical Sciences, P.O. Box 3000, FIN-90014 University of Oulu, Finland \\ 2 Tartu Observatory, 61602 Tõravere, Estonia
}

Received June 15; accepted August 3, 2000

\begin{abstract}
The method of three stage weighted period analysis is generalized for simultaneous multichannel observations. The new method is based on the phase dispersion minimization over all channels simultaneously. The three stages in the full algorithm are phase dispersion minimization method (PDM), linear modeling (LM) and nonlinear modeling (NLM). Simple computational tests of the multichannel period analysis (MPA) are presented. We show that the method has advantages if compared with methods where single channels are analysed separately. We derive a general formula for spurious periods and demonstrate that the interpretation of peaks in the PDM spectra can be more complicated than in classical power spectra.
\end{abstract}

Key words: method: analytical — data analysis

\section{Introduction}

Astronomical photometric observations are often obtained through different passbands (channels) simultaneously or quasi-simultaneously. As a rule, astronomers perform time series analysis for each observed channel separately. Sometimes normalization is used to put the channels on an equal footing (see for instance Jetsu \& Pelt 1999) and sometimes the results from different channels are averaged (You 1999). It is quite clear that in this way part of the information in the data is lost, especially if the light curves and error estimates for separate channels are substantially different. If we assume (and this is a quite reasonable assumption for most variable stars) that the periods we are seeking are the same in different passbands then we can analyze all data together using combined statistics.

$\overline{\text { Send offprint requests to: }}$ J. You, e-mail: jyou@paju.oulu.fi
The new method described in detail below is a simple and straightforward generalization of the multistage methods (Pelt 1980, 1983, 1993; Jetsu \& Pelt 1999). It is based on the phase dispersion minimization method (PDM) at the first (pilot search) stage, a linear model fit (LM) at the second (grid search) stage and finally a nonlinear model fit (NLM) at the last (refined search) stage. The first stage allows the detection of probable periods in a wide range of possible periods using fast algorithms on the coarse grid of trial frequencies. Computed periods can then be analysed to identify spurious periods which occur due to periodicities in the observation time points (Tanner 1948). Candidates which remain in the list of probable periods are then used as input for the second stage of analysis.

The LM is an intermediate stage, where refined period values are computed using equispaced high resolution grids of trial frequencies around period estimates obtained from the first stage. The purpose of the LM is to provide best initial values and correct value bracketing (search range for periods) for the final refinement.

The third stage (NLM) is in essence a nonlinear minimization procedure where the best estimates for linear model parameters (amplitudes of harmonic functions of different orders in the model) and nonlinear model parameters (periods) are obtained. It is now assumed that the initial period values for minimization and corresponding search brackets are already so well known that the convergence to a unique minimum is guaranteed. In our case of single period analysis there is only one nonlinear parameter and we can use the classical Brent minimization algorithm (Brent 1973) combined with a linear fit of the amplitudes for every particular period. The error estimate of the final value of the period can be computed from the curvature of the $\chi^{2}$ hypersurface or by using other standard methods.

The purpose of this paper is to demonstrate the usefulness of our three stage weighted multichannel period analysis (MPA) where all the available information in the input data sets are used fully and uniformly. The paper 
is organized as folllows. First we describe the MPA method in full mathematical detail in Sect. 2. Then, in Sect. 3, we apply the MPA to two groups of artificially generated data and show how all the three period searching stages help to recover the correct period. The test cases reveal the principal advantages of the new method. The results are briefly summarized in Sect. 4 .

\section{Methods}

A time series of photometric observations can be given as $y^{c}\left(t_{i}\right), \quad \sigma^{c}\left(t_{i}\right), \quad c=1,2, \ldots, C, \quad i=1,2, \ldots, N$.

$y^{c}\left(t_{i}\right)$ is the observed value of the $i$-th point in the channel $c, \sigma^{c}\left(t_{i}\right)$ is its given error, $N$ is the total number of observations and $C$ is the number of channels. The statistical weights $w_{i}^{c}$ can be computed from the errors as

$w_{i}^{c}=\frac{1}{\sigma^{c}\left(t_{i}\right)^{2}}$.

Missing points (from some channels) can be properly accounted for by using zero statistical weights.

\subsection{Multichannel PDM}

An ideal method for a preliminary period search should be independent on any assumptions about the exact shape of the light curves and the value of the periods, and be able to detect existing periods on any time scale. The multichannel PDM is devised to achieve this.

For any two observations $y^{c}\left(t_{i}\right)$ and $y^{c}\left(t_{j}\right)$, their time difference $t_{i j}$, the corresponding weight $w_{i j}^{c}$ and the dispersion contribution $y_{i j}^{c}$ are

$$
\begin{aligned}
t_{i j} & =\left|t_{i}-t_{j}\right|, \\
w_{i j}^{c} & =\frac{1}{\sigma^{c}\left(t_{i}\right)^{2}+\sigma^{c}\left(t_{j}\right)^{2}}=\frac{w_{i}^{c} \cdot w_{j}^{c}}{w_{i}^{c}+w_{j}^{c}}, \\
y_{i j}^{c} & =w_{i j}^{c} \cdot\left|y_{i}^{c}-y_{j}^{c}\right|^{2} .
\end{aligned}
$$

Here, for some time points $t_{i}$, the observations $y^{c}\left(t_{i}\right)$ may not exist for all channels. Thus, the corresponding dispersion contributions and weights are set to zero.

For a real period, a correlation exists between the proximity of the phases of two observations and the proximity of the observed values $y^{c}$. For an arbitrarily chosen period $P$ this correlation is much less probable.

The phase dispersion $D(P)$ can discriminate between these two cases:

$D(P)=\frac{\sum_{c=1}^{C} \sum_{i=1}^{N-1} \sum_{j=i+1}^{N} g\left(t_{i j}, P\right) L\left(t_{i j}\right) y_{i j}^{c}}{\sum_{c=1}^{C} \sum_{i=1}^{N-1} \sum_{j=i+1}^{N} g\left(t_{i j}, P\right) L\left(t_{i j}\right) w_{i j}^{c}}$.

Here, note that $y_{i j}^{c}$ has already the weights included according to Eq. (4). $D(P)$ is the total weighted and normalized sum of all dispersion contributions for all pairs of observations over all the channels for a trial period $P$; the additional weight component $g\left(t_{i j}, P\right)$ measures the proximity of the two observations in the phase-process diagram (PPD) computed with the trial period $P ; L\left(t_{i j}\right)$ allows us to exclude those pairs of observations which are too far from or too near to each other in time (see below).

Our particular choice for $g\left(t_{i j}, P\right)$ is

$g\left(t_{i j}, P\right)= \begin{cases}1, & \phi_{i j}(P) \leq \tau \quad \text { or } \\ & \phi_{i j}(P)>1-\tau \\ 0, & \text { otherwise. }\end{cases}$

where the relative phases $\phi_{i j}$ are defined as

$\phi_{i j}(P)=\operatorname{Frac} \frac{t_{i}-t_{j}}{P}$.

The small fixed value $\tau>0$ determines the maximum distance in phase by which $t_{i}$ and $t_{j}$ can be paired for computing $D(P)$. The smaller the value of $\tau$, the lower the number of pairs taken into account. If we compare the PDM with the standard Fourier analysis we can consider our PDM spectrum with $\tau=0.25$ as a relatively good approximation to the standard power spectrum (with another scaling). Thus, it will be most suitable for detecting single harmonics in the light curve, for which the minima in $D(P)$ correspond to the maxima in the standard power spectrum.

However, reducing $\tau$ enables us to detect more complex waveforms. At the same time, the total number of pairs taken into account in the dispersion estimate decreases and $D(P)$ becomes more erratic. Thus, the value of $\tau$ is somewhat restricted by the amount of available data points.

The selection criterion $L\left(t_{i j}\right)$ is introduced for pairing $t_{i}$ and $t_{j}$ according to their distance in time. $L\left(t_{i j}\right)$ is defined as

$L\left(t_{i j}\right)= \begin{cases}1, & D_{\min } \leq\left|t_{i}-t_{j}\right|<D_{\max } \\ 0, & \text { otherwise }\end{cases}$

The degree of smoothness of the $D(P)$ spectrum obtained by the use of $L\left(t_{i j}\right)$ depends on $D_{\max }$. When $D_{\max }=$ $t_{N}-t_{1}$, all pairs are taken into account when computing $D(P)$, and the maximal resolution is attained. For a smaller value of $D_{\max }$, only a subset of pairs is considered and the computed $D(P)$ is smoother. This allows us to compute rough PDM spectra with a larger step in frequency. For long data sets the computing time advantage can be significant. A typical choice of $D_{\max }$ depends on the maximum trial period $P_{\max }$, e.g. $D_{\max } \geq 10 \cdot P_{\max }$. It also depends on the actual distribution of the time points.

If the time points $t_{i}$ and $t_{j}$ are too close, i.e. $\left|t_{i}-t_{j}\right|$ is less than the minimal trial period $P_{\text {min }}$, the corresponding terms in $D(P)$ do not change significantly for all trial periods so that it does not help to discriminate between them. To avoid unnecessary computations, the lower limit $D_{\text {min }}$ discards such pairs from the sum of $D(P)$. A typical choice of $D_{\min }$ depends on the minimum trial period $P_{\min }$, e.g. $D_{\min }=0.9 \cdot P_{\min }$.

The straightforward computation of the $D(P)$ spectra can be very time consuming. Following a simple binning scheme allows us to speed up calculations significantly. 
We divide the full range of $D_{\min } \leq t_{i j}<D_{\max }$ into

$K=\operatorname{Int}\left(\frac{D_{\max }-D_{\min }}{\Delta}\right)+1$

bins of equal width $\Delta$ ( $K \ll N$ for a large data set). The bin width can be chosen as $\Delta=0.1 \times P_{\min }$. Then, we denote the $n_{q}$ values of $t_{i j}, w_{i j}{ }^{c}$ and $y_{i j}{ }^{c}$ in the $q$-th bin as $t_{q, k}, w_{q, k}^{c}$ and $y_{q, k}^{c}$ and derive

$\bar{t}_{q}=\frac{\sum_{k=1}^{n_{q}} t_{q, k}}{n_{q}}$,

$\bar{y}_{q}^{c}=\sum_{k=1}^{n_{q}} y_{q, k}^{c}$,

$\bar{w}_{q}^{c}=\sum_{k=1}^{n_{q}} w_{q, k}^{c}$.

Note that $y_{q, k}^{c}$ has already been weighted according to Eq. (4). It is important that these sums do not depend on the trial periods and consequently can be precomputed. The final modified sum for $D(P)$ over all channels is

$\bar{D}(P)=\frac{\sum_{c=1}^{C} \sum_{q=1}^{K} g\left(\bar{t}_{q}, P\right) \bar{y}_{q}^{c}}{\sum_{c=1}^{C} \sum_{q=1}^{K} g\left(\bar{t}_{q}, P\right) \bar{w}_{q}^{c}}$,

and it can be computed reasonably fast even for quite large data sets.

In this first search stage we only identify a set of approximate period candidates for further refinement and identification. Small approximation errors which result from the binning scheme do not hide significant peaks in the PDM spectra. The PDM stage is most useful in the situations where the full time span of the data set is large compared with expected period. For short data sets it can be skipped altogether.

\subsection{Multichannel linear modeling}

In the second stage of the period search we build regular search grids around every period candidate found in the PDM stage. Minima from these short LM spectra with the full resolution are then used as starting values for the nonlinear full precision fit.

We compute the second stage LM spectra using a weighted least squares fit of the model curve $M(t, \beta(P))$ in the form of trigonometric sums (Koen 1999):

$$
\begin{aligned}
& M(t, \beta(P)) \\
& =A_{0}+\sum_{r=1}^{R}\left[A_{r} \cos (2 \pi r f t)+B_{r} \sin (2 \pi r f t)\right],
\end{aligned}
$$

where $f=\frac{1}{P}$ is the trial frequency and $\beta(P)$ includes all linear parameters $A_{0}, A_{r}, B_{r}$. We choose the order $R$ for the trigonometric polynomial according to $\tau$ chosen in the PDM search. A general relation is $4 R \tau=1$, e.g. $R=1$ for $\tau=0.25, R=2$ if $\tau=0.125$, etc. Note that the choice of $\tau$ depends on the complexity of the real light curve and the number of data points, thus, the choices of $R$ and $\tau$ should be consistent.
The step size for the frequency grids is computed from $\Delta f_{\mathrm{LM}}=\frac{1}{G \cdot\left(t_{\max }-t_{\min }\right)}$,

where $G$ is a scaling factor which depends on the order of the fit. LM spectra for single harmonic models can be computed on coarser grids. From theory we know (using the Nyquist criterion) that $G=2$ is good enough, however, to be on the safe side, we normally can use modest oversampling $(G=2.5 \cdot 2=5)$. For higher order trigonometric fits the Nyquist criterion must be applied for the highest frequency in the model and correspondingly the choice $G=2.5 \cdot 2 \cdot R$ is a good choice.

The last important parameter of the grid search is the frequency range. For every candidate period $P_{\mathrm{PDM}}=$ $\frac{1}{f_{\mathrm{PDM}}}$, we define a frequency grid around it spanning at least $\left[f_{\mathrm{PDM}}-5 \Delta f_{\mathrm{PDM}}, f_{\mathrm{PDM}}+5 \Delta f_{\mathrm{PDM}}\right]$, where $\Delta f_{\mathrm{PDM}}$ is the step size in the PDM search. In this way the approximate periods (frequencies) from the first search stage are allowed to contain rather large errors. Relatively wide ranges for the second stage search are important also in situations where multiple, narrowly spaced peaks blend into one in smooth spectra.

The weighted residual sums of squares of a single channel

$W R S S^{c}(P)=\sum_{i=1}^{N} w_{i}^{c}\left[y_{i}^{c}-M\left(t_{i}, \beta^{c}(P)\right)\right]^{2}$,

are combined into

$W R S S(P)=\sum_{c=1}^{C} W R S S^{c}(P)$,

to get short spectra around each candidate period. The period values which correspond to the most significant peaks in these spectra form a set of period candidates for a final refinement.

\subsection{Multichannel nonlinear modeling and error estimation}

The precision of the periods obtained from the grid search is limited by the frequency step used. In the third stage we allow trial frequencies to vary continuously, while seeking the minimum of WRSS (Eq. (17)). Here, the period $P$ is treated as the single nonlinear parameter, which determines (through linear weighted least squares estimations) the entire group of parameters $\beta(P)$ (see Eq. (14)). The well-known Brent minimization algorithm (Brent 1973; Chapter 10 in Press et al. 1994) can now be used to find the local minimum of $W R S S(P)$. For each trial frequency we need to choose proper brackets for the minimization. To do that, we must carefully inspect LM spectra obtained in the second stage of the search. However, in most cases the rule of thumb "five steps left, five steps right" works reasonably well. 
As a result of weighted linear least squares fit (for a particular frequency) we get the values and the error estimates for the linear parameters $\beta(P)$ (Chapter 15 in Press et al. 1994). The error estimates are valid only in the case when the frequency is assumed to be accurate, i.e. the correlations between the linear parameters and the period are not taken into account. Koen (1999) has pointed out that these error estimates for the linear parameters are different from those obtained when the errors are estimated together with the frequency error.

For the nonlinear parameter $P$, the error estimate $\sigma_{P}$ of the final period relies on the curvature of the $W R S S(P)$ hypersurface (Chapter 11 in Bevington 1969), which is

$\sigma_{P}^{2}=\frac{2}{\partial^{2} \chi^{2} / \partial P^{2}}$.

Here, $\chi^{2}$ is identical to the WRSS in Eq. (17). The error estimate $\sigma_{P}$ is reasonably correct only if the variation of $\chi^{2}$ with respect to the nonlinear parameter $P$ is independent of the values of all the linear parameters $\beta(P)$ (at least near the minimum) and the observation errors in the different channels are uncorrelated and correctly estimated.

After Brent's algorithm traced the minimum of the $W R S S$ function, the parabolic curve fitted to the last three points of $(P, W R S S(P))$ is used for the actual computation of $\sigma_{P}^{2}$.

\subsection{Spurious periods}

The approximate periodicities in the observing times reveal themselves as a series of spurious periods in the classical power spectra (Deeming 1975) as well as in the PDM spectra (Tanner 1948). The general expression for the spurious periods originating from the interplay of a correct period $P$ with periodicities in the observing times is:

$\frac{r}{P_{r, l, s_{1}, s_{2}, \ldots, s_{K}}}=\frac{l}{P}+\sum_{k=1}^{K} \frac{s_{k}}{\delta_{k}}$,

where integers $r=1,2, \ldots, R, l=0, \pm 1, \pm 2, \ldots, s_{k}=$ $0, \pm 1, \pm 2, \ldots, k=1,2, \ldots, K$ are constrained by the condition $P_{r, l, s_{1}, s_{2}, \ldots, s_{K}}>0$ and the $\delta_{k}$ s are the $K$ periods in the time point spacing. Equation (19) can be derived as follows.

Assume for the moment that the observing times $t_{i}$ can be exactly expressed as

$t_{i}=n_{i} \cdot \delta_{k}+t_{1}, \quad i=1,2, \ldots, N$,

where $n_{i}$ are integer numbers and $\delta_{k}$ is a particular period in the data spacing. In astronomical observations, the typical periods are the sidereal day, the tropical year and the lunar month. The actual data spacing periods can be found from the transformed data window (see Deeming 1975).

Two observations at times $t_{i}, t_{j}$ have the same phase in the PPD when $t_{j}-t_{i}=a_{i, j} \cdot P$ for some integer number $a_{i, j}$. If these points are from an equally spaced mesh with period $\delta_{k}$ then for some other integer number $b_{i, j, k}$ we have $t_{j}-t_{i}=b_{i, j, k} \delta_{k}$. We can now combine the integer numbers $a_{i, j}$ and $b_{i, j, k}$ into various integer sums

$c_{i, j}=l \cdot a_{i, j}+\sum_{k=1}^{K} s_{k} \cdot b_{i, j, k}$,

where $l$ and $s_{k}$ are arbitrary integer multipliers. Using the above definitions

$c_{i, j}=\frac{l\left(t_{j}-t_{i}\right)}{P}+\sum_{k=1}^{K} \frac{s_{k}\left(t_{j}-t_{i}\right)}{\delta_{k}}$,

we see that from the closeness of the phases for the correct period $P$ follows the closeness of the corresponding phases for a full series of other periods

$t_{j}-t_{i}=P_{l, s_{1}, s_{2}, \ldots, s_{K}} \times c_{i, j}$

where

$P_{l, s_{1}, s_{2}, \ldots, s_{K}}=\left(\frac{l}{P}+\sum_{k=1}^{K} \frac{s_{k}}{\delta_{k}}\right)^{-1}$.

Because all phase dispersion estimates depend on the period through the phases, we can now conclude: if for a certain period $P$, the PPD shows a small amount of scatter and there is a corresponding peak in the PDM spectrum, then, for all spurious periods $P_{l, s_{1}, s_{2}, \ldots, s_{K}}$ we can see a similar picture. Because the periodicities in the time point spacings are only approximate, the actual picture is somewhat more complicated. The PPDs for spurious periods tend to have larger scatter when compared with the diagram for the correct period. It is important to note that spurious periods are not artifacts of a certain period search method. They occur universally when there are regularities in the data spacing.

There is an integer index $r$ in Eq. (19) which needs additional explanation. So far, we explained how one particular period $P$ will produce "ghosts" in the spectra if the data time points contain periodicities. Our simple explanation in terms of phase relations can be complemented by a detailed analysis using Fourier transforms as it is done in Deeming (1975). If we compare PDM spectra (or LM spectra with the order $R$ chosen according to $\tau$ ) and Fourier spectra, we see that PDM (or LM) spectra, which are computed using multi-harmonic fits, contain many more spurious peaks than the Fourier spectra. Only if we compute PDM (or LM) spectra for a single harmonic model do we get the same picture as in the standard power spectra.

The difference comes from the fact that phasedispersion estimators for one particular trial period $P$ depend also on higher harmonics $P / r$, where $r=1,2, \ldots$. For instance, in the particular case of a two-harmonic LM fit, it is quite obvious that the resulting dispersion for the trial periods depends at least on the spectral power at $P$ and at $P / 2$. This shows up in a quite straightforward way. In the spectrum (computed using a two-harmonic fit), we can see not only the strong minimum for the correct period $P$ (and possibly one at $P / 2$ ) but also a strong 
Table 1. Model amplitudes and error levels (Eqs. $(25,26))$ for the first group of simulations

\begin{tabular}{ccccccc}
\hline$c$ & $A_{0}^{c}$ & $A_{1}^{c}$ & $B_{1}^{c}$ & $A_{2}^{c}$ & $B_{2}^{c}$ & $E^{c}$ \\
\hline$U$ & 0.0 & 0.9 & 0.4 & 0.5 & 0.4 & 3.0 \\
$B$ & 0.0 & 0.8 & -0.5 & 0.6 & 0.3 & 2.5 \\
$V$ & 0.0 & -0.7 & 0.6 & 0.7 & 0.2 & 2.0 \\
$R$ & 0.0 & -0.6 & -0.7 & 0.8 & 0.1 & 1.5 \\
\hline
\end{tabular}
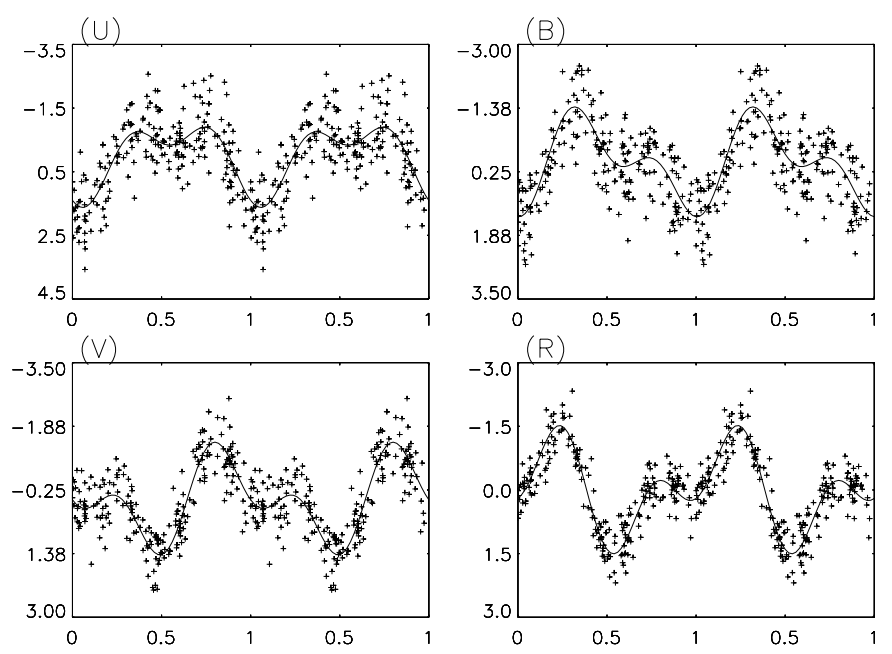

Fig. 1. PPDs of $U, B, V$ and $R$ of set 3 in the first group of simulations. The noise level is $0.25 E^{c}$ (see Table 3 ). The $E^{c}$ s are fixed for all observations in a particular channel (see Table 1)

minimum for $2 P$, which results from the fit of only the second harmonic to the part of the light curve generated by the first harmonic. The PDM (or LM) spectrum detects periods, not harmonics. If the process is periodic with period $P$, then it is periodic also with period $2 P$ ! As a result of this, all spurious periods belonging to every real harmonic in the spectrum will generate additional ghosts around the multiples of periods in the spectra.

Formally we take this into account using the index $r$ in Eq. (19). The set of spurious periods given by Tanner (1948) is the special case for $r=1, K=1$ in Eq. (19). From Eq. (19) we can compute only the positions of potential spurious peaks in the spectrum. The actual strengths of the peaks depend on the harmonic content of the process itself, on the time point distribution etc. (see for instance, the identification of spurious periods of the set 5 from the second group of simulations in Sect. 3). It is also possible that some of the spurious periods can constructively or destructively interfere with each other. This is especially true for multiperiodic light curves (see Pelt 1997).

\section{Simple tests}

We tested MPA with two groups of artificially generated data. For both data groups we used a light curve model of two harmonics

$$
\begin{aligned}
M^{c}\left(t_{i}\right)= & A_{0}^{c}+A_{1}^{c} \cos \left(\frac{2 \pi t_{i}}{P}\right)+B_{1}^{c} \sin \left(\frac{2 \pi t_{i}}{P}\right) \\
& +A_{2}^{c} \cos \left(\frac{4 \pi t_{i}}{P}\right)+B_{2}^{c} \sin \left(\frac{4 \pi t_{i}}{P}\right)+\epsilon_{i}^{c} .
\end{aligned}
$$

Specific parameters for different models were chosen as follows. Time points $t_{i}, i=1, \ldots, N$ (where the number of observations $N=211$ ) were obtained from the yet unpublished photometric data set of the RS CVn binary ER Vul obtained at Mount Maidanak Observatory in 1990-1996 with the $60 \mathrm{~cm}$ telescope. The typical errors for this $U B V R$ photometry are about 0.030 for the $U$ channel and 0.015 for the others (for details see Shevchenko 1980). In this way, the distribution of time points for our simulations contains complexities which are characteristic for real observational sequences. For all the experiments described here, we used the fixed period $P=0.6980993$, which comes from our preliminary analysis of the ER Vul data. In the first group of the experiments, we chose amplitudes $A_{r}^{c}$ and $B_{r}^{c}$ arbitrarily (see Table 1 and Fig. 1) to get a rather complex waveform as the light curve.

In the second group of experiments we used the parameters of a two-harmonic fit to the real light curve of ER Vul, which were estimated previously (You 1999). The corresponding amplitudes and error levels are given in Table 2 and the light curves are shown in Fig. 2. The most important difference between the two groups of simulations is the relatively low amplitude of the first harmonic in the second group.

Finally, to the computed trigonometric polynomials we added normally distributed uncorrelated noise $N_{i}(0,1)$ which we computed using a random number generator:

$\epsilon_{i}^{c}=a \cdot E^{c} \cdot N_{i}(0,1)$,

where $a$ is a noise amplification factor which we choose to be in the range $0.01-10$ and $E^{c}$ are the error levels in different channels $(c=1,2,3,4)$.

To test the new method, both the multichannel PDM and the single channel PDM were applied for all channels of the two groups of simulations. In the first place, we wanted to compare the detection capabilities of the multichannel method to that of the single channel analysis. This is why we chose a relatively narrow period search range $P_{\min }=0.65$ and $P_{\max }=0.75$. In this way the real period buried in the noise will basically "compete" only with the random fluctuations. A more comprehensive search where spurious periods are taken into account seriously is described afterwards.

For the smoothing criterion $L\left(t_{i j}\right)$, we adopted $D_{\min }=$ $0.9 \cdot P_{\min }, D_{\max }=100 \cdot P_{\max }$ and for the selection criterion $g\left(t_{i j}, P\right)$ we set $\tau=0.125$ according to the above adopted models. These are typical values and do not depend strongly on the data to be analysed.

Tables 3 and 4 show the recovered periods $P_{\mathrm{PDM}}$ from the multichannel PDM for the first and the second groups 
Table 2. Model amplitudes and error levels (Eqs. (25) and (26)) for the second group of simulations

\begin{tabular}{lcccccc}
\hline$c$ & $A_{0}^{c}$ & $A_{1}^{c}$ & $B_{1}^{c}$ & $A_{2}^{c}$ & $B_{2}^{c}$ & $E^{c}$ \\
\hline$U$ & 8.005674 & 0.011960 & 0.001157 & -0.040586 & 0.003809 & 0.030 \\
$B$ & 7.960660 & 0.009258 & 0.002582 & -0.044870 & 0.006782 & 0.015 \\
$V$ & 7.354608 & 0.006704 & 0.002303 & -0.042520 & 0.006269 & 0.015 \\
$R$ & 6.795945 & 0.006663 & 0.002186 & -0.041944 & 0.006590 & 0.015 \\
\hline
\end{tabular}
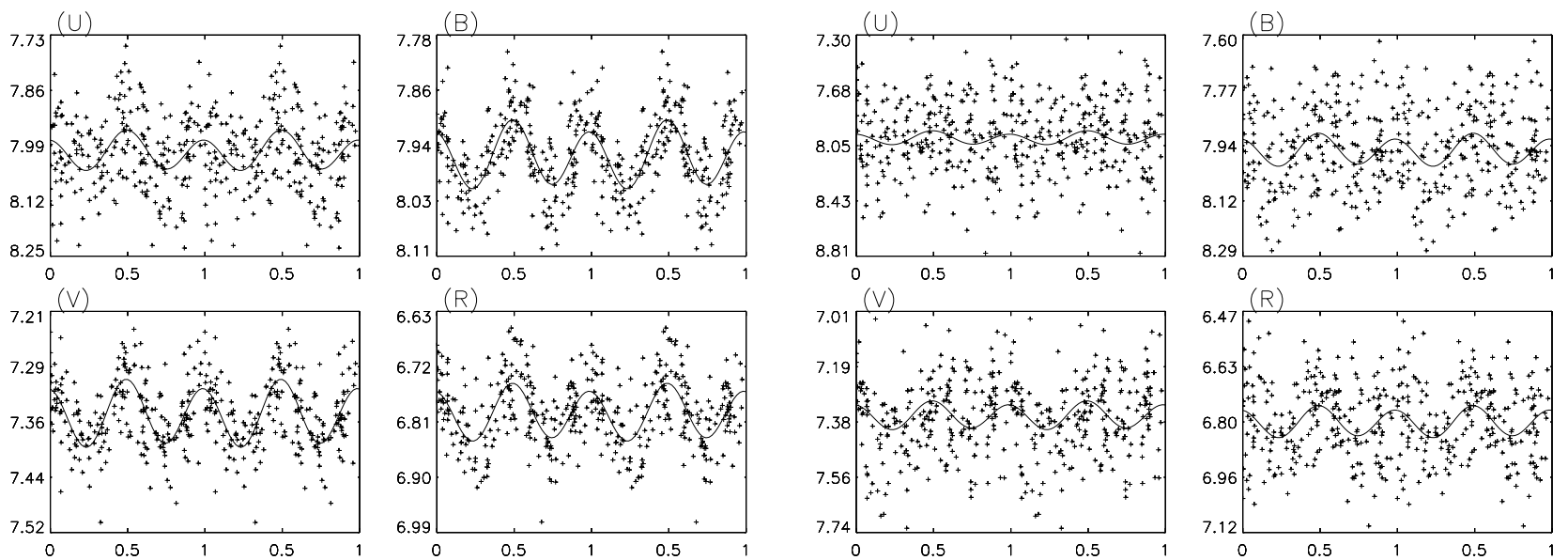

Fig. 2. PPDs of $U, B, V$ and $R$ of sets 7 (left) and 12 (right) in the second group of simulations. The corresponding noise levels are $3.0 E^{c}$ and $8.0 E^{c}$ (see Table 4 ). The $E^{c} \mathrm{~s}$ are the real estimated observation errors for $U B V R$ (see Table 2)

Table 3. Results of the three stage weighted MPA for the first group of simulations in comparison with the breakdown noise levels of the single channel search (see Sect. 3 for explanations). The true period is 0.6980993

\begin{tabular}{lrccccccc}
\hline Set & Noise & $S / N^{\mathrm{U}}$ & $S / N^{\mathrm{B}}$ & $S / N^{\mathrm{V}}$ & $S / N^{\mathrm{R}}$ & $P_{\mathrm{PDM}}$ & $P_{\mathrm{LM}}$ & $P_{\mathrm{NLM}}$ \\
\hline 1 & $0.01 E^{c}$ & 1.43 & 1.53 & 1.63 & 1.78 & 0.698 & 0.69810094 & $0.69809942 \pm 0.00000014$ \\
2 & $0.1 E^{c}$ & 0.41 & 0.49 & 0.60 & 0.77 & 0.698 & 0.69810094 & $0.6981014 \pm 0.0000014$ \\
3 & $0.25 E^{c}$ & 0.03 & 0.08 & 0.22 & 0.35 & 0.698 & 0.69809550 & $0.6980980 \pm 0.0000036$ \\
4 & $0.5 E^{c}$ & -0.25 & -0.19 & -0.05 & 0.06 & 0.698 & 0.69810094 & $0.6980991 \pm 0.0000075$ \\
5 & $0.75 E^{c}$ & $\mathbf{- 0 . 4 5}$ & -0.34 & -0.22 & -0.10 & 0.698 & 0.69810094 & $0.698099 \pm 0.000010$ \\
6 & $1.0 E^{c}$ & -0.53 & -0.49 & -0.39 & -0.23 & 0.698 & 0.69807374 & $0.698075 \pm 0.000013$ \\
7 & $1.25 E^{c}$ & -0.68 & $-\mathbf{0 . 5 7}$ & $-\mathbf{0 . 4 7}$ & -0.36 & 0.698 & 0.69809006 & $0.698088 \pm 0.000019$ \\
8 & $1.5 E^{c}$ & -0.75 & -0.65 & -0.55 & -0.40 & 0.697 & 0.69811130 & $0.698109 \pm 0.000020$ \\
9 & $1.75 E^{c}$ & -0.79 & -0.71 & -0.57 & $-\mathbf{0 . 4 8}$ & 0.697 & 0.69810586 & $0.698108 \pm 0.000032$ \\
10 & $2.0 E^{c}$ & -0.84 & -0.82 & -0.65 & -0.53 & 0.698 & 0.69816081 & $0.698162 \pm 0.000029$ \\
11 & $2.25 E^{c}$ & -0.88 & -0.83 & -0.72 & -0.58 & 0.697 & 0.69816570 & $0.698166 \pm 0.000037$ \\
12 & $2.5 E^{c}$ & -0.94 & -0.88 & -0.76 & -0.66 & - & - & \\
\hline
\end{tabular}

Table 4. Results of the three stage weighted MPA for the second group of simulations (see Sect. 3 for explanations)

\begin{tabular}{lrccccccc}
\hline Set & Noise & $S / N^{\mathrm{U}}$ & $S / N^{\mathrm{B}}$ & $S / N^{\mathrm{V}}$ & $S / N^{\mathrm{K}}$ & $P_{\mathrm{PDM}}$ & $P_{\mathrm{LM}}$ & $P_{\mathrm{NLM}}$ \\
\hline 1 & $0.01 E^{c}$ & 1.75 & 1.98 & 1.92 & 1.91 & 0.698 & 0.69809688 & $0.698099296 \pm 0.000000023$ \\
2 & $0.1 E^{c}$ & 0.96 & 1.33 & 1.29 & 1.27 & 0.698 & 0.69809688 & $0.69809935 \pm 0.00000023$ \\
3 & $0.25 E^{c}$ & 0.61 & 0.97 & 0.91 & 0.90 & 0.698 & 0.69810232 & $0.69810025 \pm 0.00000057$ \\
4 & $0.5 E^{c}$ & 0.32 & 0.66 & 0.60 & 0.61 & 0.698 & 0.69809688 & $0.6980982 \pm 0.0000011$ \\
5 & $1.0 E^{c}$ & -0.01 & 0.32 & 0.28 & 0.31 & 0.698 & 0.69809688 & $0.6980982 \pm 0.0000022$ \\
6 & $2.0 E^{c}$ & $-\mathbf{0 . 3 1}$ & 0.02 & -0.02 & 0.02 & 0.698 & 0.69809688 & $0.6980978 \pm 0.0000042$ \\
7 & $3.0 E^{c}$ & -0.45 & -0.14 & $\mathbf{- 0 . 1 2}$ & $-\mathbf{0 . 1 9}$ & 0.698 & 0.69810232 & $0.6981013 \pm 0.0000068$ \\
8 & $4.0 E^{c}$ & -0.66 & -0.24 & -0.27 & -0.31 & 0.698 & 0.69810776 & $0.698108 \pm 0.000010$ \\
9 & $5.0 E^{c}$ & -0.71 & $-\mathbf{0 . 3 5}$ & -0.39 & -0.39 & 0.698 & 0.69811321 & $0.698111 \pm 0.000013$ \\
10 & $6.0 E^{c}$ & -0.75 & -0.44 & -0.44 & -0.50 & 0.698 & 0.69808464 & $0.698083 \pm 0.000013$ \\
11 & $7.0 E^{c}$ & -0.83 & -0.53 & -0.53 & -0.53 & 0.698 & 0.69810097 & $0.698100 \pm 0.000019$ \\
12 & $8.0 E^{c}$ & -0.92 & -0.59 & -0.60 & -0.55 & 0.698 & 0.69811865 & $0.698116 \pm 0.000018$ \\
13 & $9.0 E^{c}$ & -0.95 & -0.64 & -0.63 & -0.67 & 0.698 & 0.69810232 & $0.698104 \pm 0.000018$ \\
14 & $10.0 E^{c}$ & -0.97 & -0.67 & -0.70 & -0.72 & - & - & \\
\hline
\end{tabular}




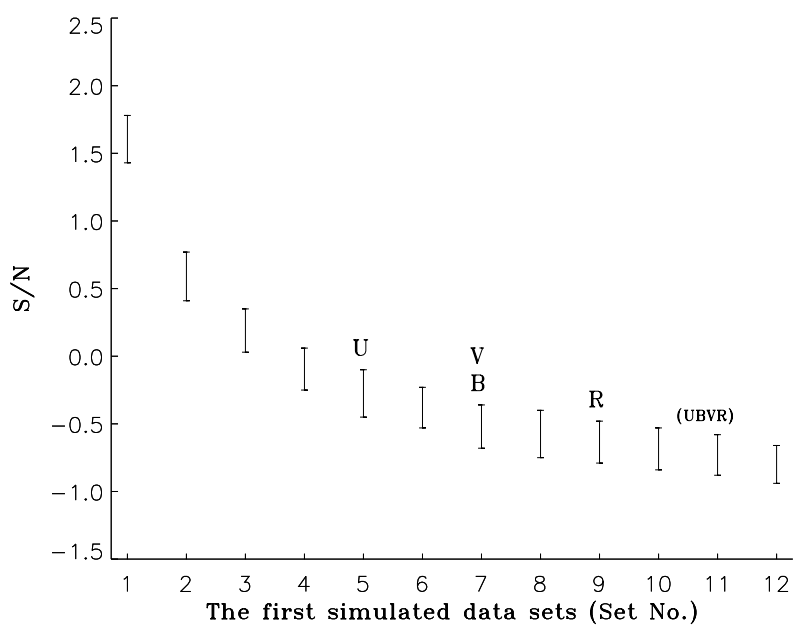

Fig. 3. Detection levels in the PDM search for the first group of simulations. Vertical bars denote the $S / N$ range over four channels. The last successful detections are marked with $U, B$, $V$ and $R$ for computations of a single channel and with $U B V R$ for the multichannel method

of simulations, respectively. As a noise level indicator, we define a logarithmic signal to noise ratio as

$S / N^{c}=\log \left(\frac{\sigma_{m}^{c}}{\sigma_{n}^{c}}\right), \quad c=1,2,3,4$

where $\sigma_{m}^{c}$ is the standard deviation of the model data points around the mean and $\sigma_{n}^{c}$ is the standard deviation of the simulated data points around the model curve. $\sigma_{m}^{c}$ is chosen for two reasons: (1) The true amplitude of the model curve would be a natural choice, however, for complex shapes with many harmonics the definition of the amplitude is not straightforward; (2) We are dealing with only the discrete time points and there is no guarantee that the true maximum/minimum of the model curve appears among these points. When the noise level increases, the $S / N^{c}$ decreases. Starting from a certain noise level, the correct peak in the spectrum will be less deep than some of the nearby random fluctuations. We marked in the Tables 3 and 4 with boldface those noise levels where the correct peak occured for the last time as the strongest minimum. It is well seen that the multichannel version of the spectrum reveals the true period for higher noise levels compared to the single channel spectra. Due to the random nature of the simulations and different noise levels in the different channels, the breakdown noise levels cannot be predicted precisely using analytical expressions.

In Figs. 3 and 4, these results are depicted graphically. We can see the clear difference between detection levels for the PDM search. The main peak in the spectra for the $U, B, V$ and $R$ channels "sinks" into the noise significantly earlier than the main peak in the combined spectrum. As an illustration, we depict some actual spectra in Figs. 5 and 6 . Here, it needs to be remarked that the deepest minimum at 0.698 in the $(V)$ spectrum of Fig. 6 was produced accidentally by random fluctuations in the data

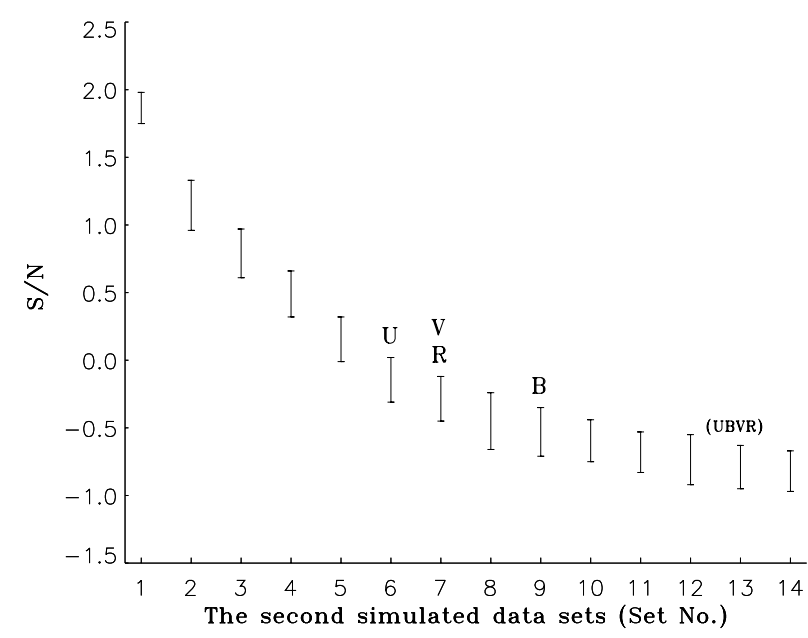

Fig. 4. Detection levels in the PDM search for the second group of simulations. For details, see Fig. 3

set 12 . In the second group of simulations, the $V$ channel has already stopped working from the set 7 (Fig. 4), for which Fig. 5 shows its last correctly working spectrum.

Our simple tests with the restricted period search range well demonstrate that the expected improvement in precision and in period detection capability indeed takes place.

For a wider search range this nice picture is spoilt by spurious periods. We applied PDM for the range of periods $P_{\min }=0.3$ and $P_{\max }=1.9$ to obtain the single channel and the combined spectra which are depicted in Fig. 7. All five spectra show the four most significant minima at periods $0.349,0.537,1.161$ and 0.698 . The correct period $P=0.698$ is always detected but it is far from the best one! If we look at the corresponding data window which is computed as in Deeming (1975), we see that our data spacing is nearly periodic with $\delta=0.99728738$ and consequently every peak in the spectrum is echoed multiple times. Using Eq. (19), we can identify spurious periods which are actually echoes of the real period. We summarize the results of the identification of all the nine spurious periods shown in Fig. 7 in Table 5.

The spurious period of 0.349 with the deepest minimum occurs at the location where $P_{1,2,0}=0.34904965$; the spurious period 0.537 with the second deepest minimum occurs at the location where $P_{1,2,-1}=0.53699869$ and finally the spurious period of 1.161 with the third deepest minimum occurs at $P_{1,2,-2}=1.1634916$. Because the amplitudes of the second sinusoidal function is much larger than those of the first one in the simulated model (Table 2), the model curve resembles that of only one sinusoidal function with half the correct period. Thus, this results in the large significance of the first three spurious periods and also for $P_{2,2,-1}$ and $P_{2,2,1}$ which occur due to fitting with only the $(r=2)$ sinusoidal function (see explanation in Sect. 2.4). 


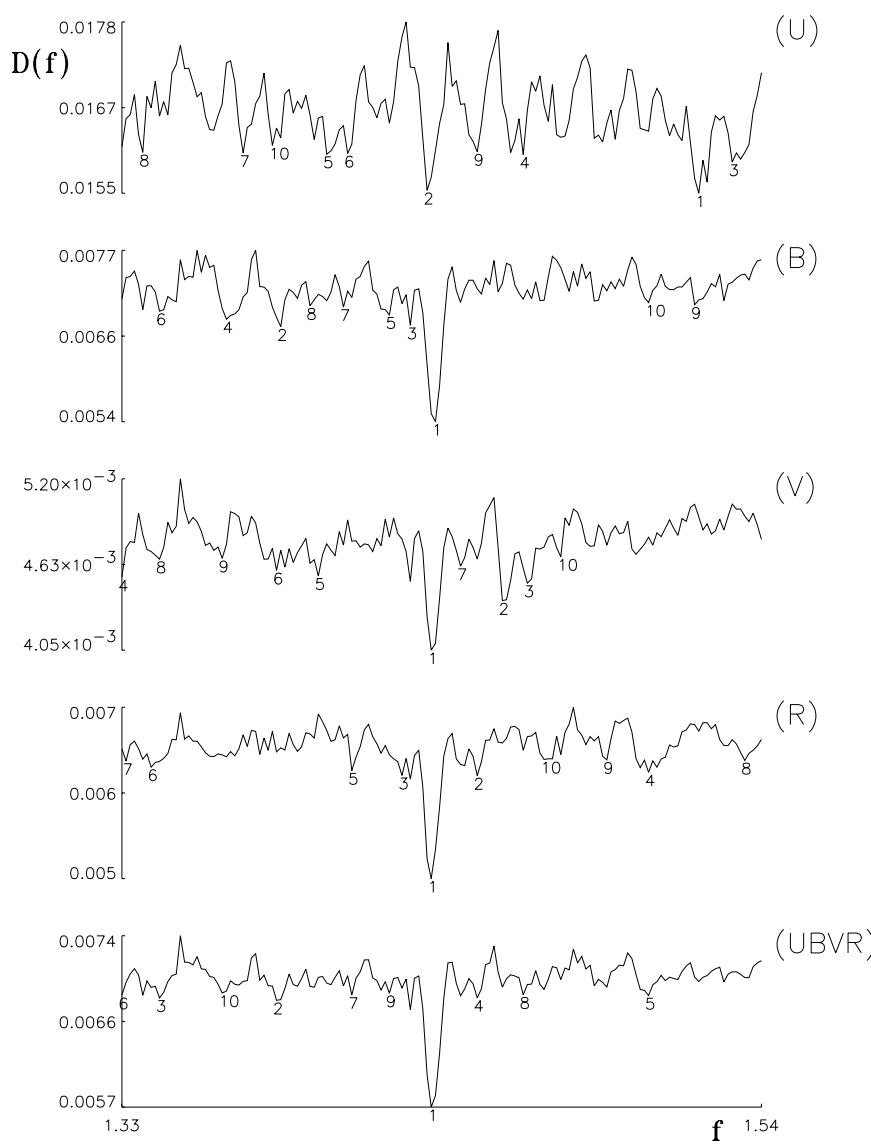

Fig. 5. Set of PDM spectra computed for the single channels and for the combined multichannel of set 7 (noise level $3.0 E^{c}$ ) in the second group of simulations. The numbers label the depth ranks of the valleys in the spectra

Table 5. Identification of spurious periods with the Deeming window of set 5 in the second group of simulations. No. is the rank of the period shown in the combined spectrum of $(U B V R)$ in Fig. 7, $P_{\mathrm{PDM}}$ is the value found with the multichannel PDM and $P_{r, l, s}$ are computed from Eq. (19) with the corresponding $(r, l, s)$ and $\delta=0.99728738$

\begin{tabular}{lccr}
\hline No. & $P_{\text {PDM }}$ & $P_{r, l, s}$ & $(r, l, s)$ \\
\hline 1 & 0.3490423 & 0.3490497 & $(1,2,0)$ \\
2 & 0.5365817 & 0.5369987 & $(1,2,-1)$ \\
3 & 1.1614638 & 1.1634916 & $(1,2,-2)$ \\
5 & 1.0733655 & 1.0739974 & $(2,2,-1)$ \\
6 & 0.5169787 & 0.5171110 & $(2,2,1)$ \\
7 & 0.8743941 & 0.8726323 & $(1,-2,4)$ \\
8 & 0.4107931 & 0.4106471 & $(1,1,1)$ \\
9 & 1.7493248 & 1.7452646 & $(1,-1,2)$ \\
10 & 0.4662043 & 0.4654024 & $(1,-2,5)$ \\
\hline
\end{tabular}

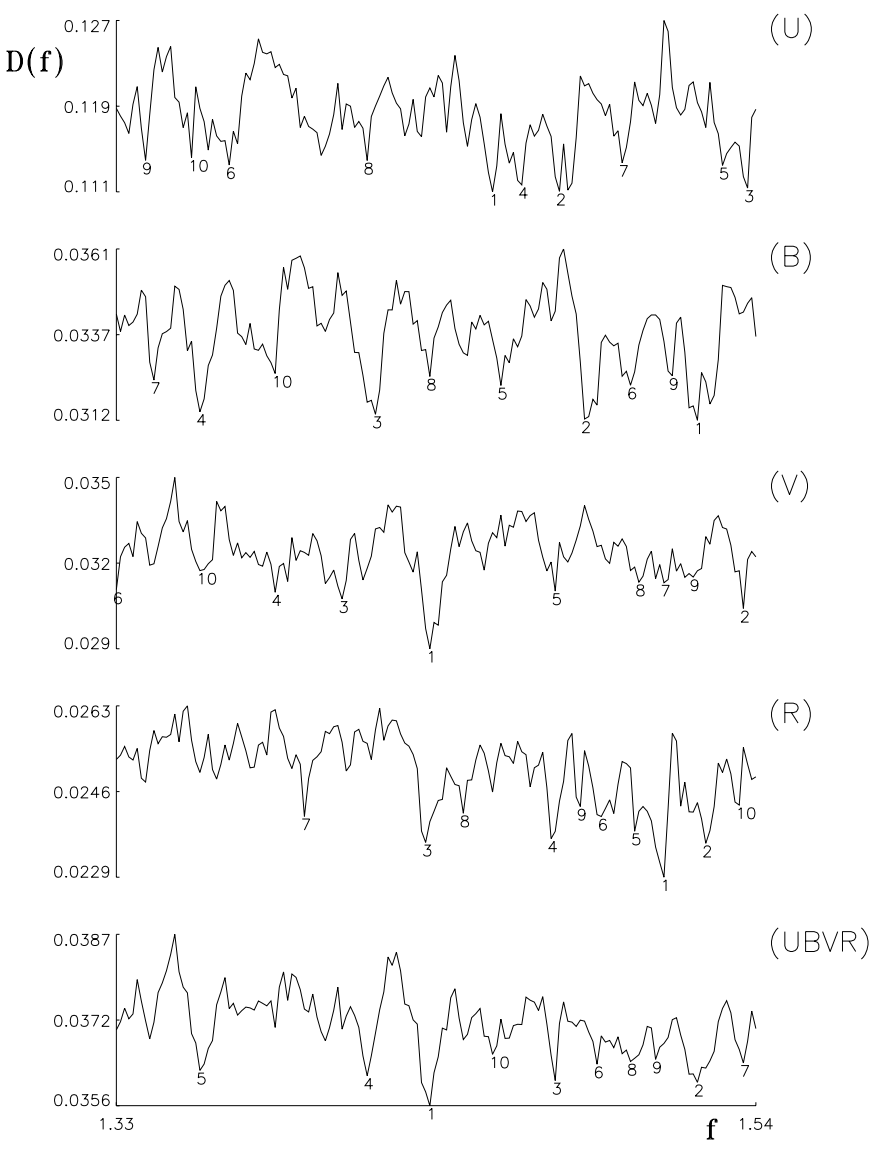

Fig. 6. Set of PDM spectra computed for the single channels and for the combined multichannel of set 12 (noise level 8.0 $E^{c}$ ) in the second group of simulations

This is a very important observation. The interplay of the real periodicity with a periodicity in the time point distribution can totally change the general appearance of the spectra. Even when we use all available information (as in the case of the multichannel search), the spurious periods can still complicate our analysis. Paradoxically enough, the improved detection capability of multichannel methods increases also the ability to detect spurious periods. So, when working with real data we must always carefully inspect the spectra using the information from the corresponding data windows to reveal the real periods.

In the last two columns of Tables 3 and 4 the refined periods obtained with LM and NLM are given. Figures 8 and 9 show the final refined periods computed with MPA for the two groups of simulations. For comparison, the results from the search using the best single channel (with highest $S / N)$ are also given (if available). We see that MPA gives as a rule more precise estimates for the main period than those from only the best single channel.

Because the differences between standard least squares methods for the single channels and for the multichannel are minimal, we are not going here into the details of computation. There are only two important additional 

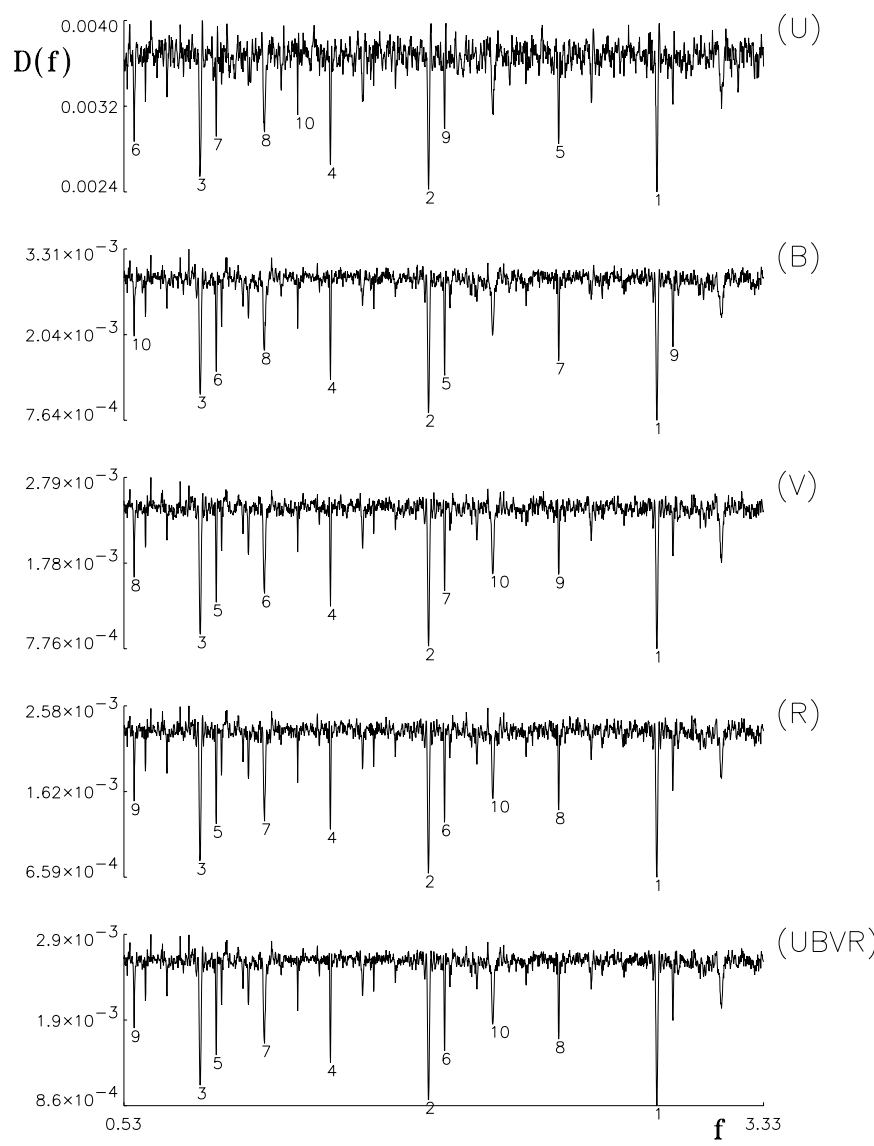

Fig. 7. PDM spectra of set 5 (noise level $1.0 E^{c}$ ) in the second group of simulations. In all five spectra the real period is fourth ranked according to the depth of a peak. All others are spurious and can be identified according to Eq. (19)

considerations to be taken into account. The first one is the correct choice of the frequency step for the LM and the correct frequency bracketing for the final NLM refinement. Because of the improved detection capability of the multichannel method, we can in principle choose somewhat larger step sizes for LM and somewhat narrower brackets for a final period improvement. This is certainly true for exact periodicities. But in real life situations, the periods can be slightly varying and, as a result of this, the minima around the periods can have less regular profiles.

The second consideration is the error estimation for the final computed periods. In the ideal case of exact periods with reasonably even sampling and full time coverage, the error estimates based on the curvature of $W R S S(P)$ can give good estimates for period errors. But for more complex cases, bootstrap type procedures must be involved (see Jetsu \& Pelt 1999). The bootstrap for multichannel methods must take into account the special structure of the input data and will only work if the residuals are uncorrelated. It is reasonable to reshuffle residuals for each channel separately with correct reweighting to ensure that the statistical structure of each bootstrap run will be analogous to the original data.

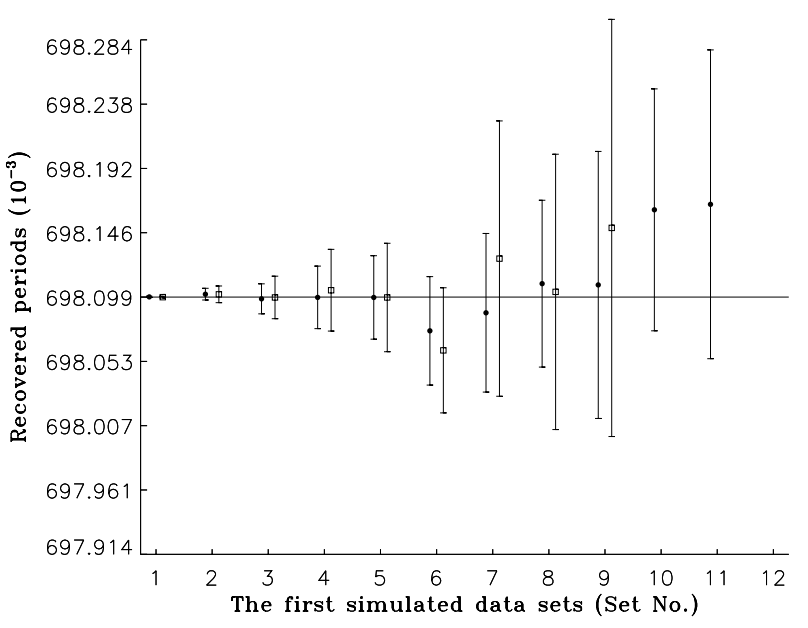

Fig. 8. Periods (filled circles) and their errors ( $3 \sigma$ bars) recovered with MPA for the first group of simulations. The $x$-axis is the data set number and the $y$-axis is the period in $10^{-3}$. The horizontal line is at the correct period 0.6980993. Additionally (if available), the best estimates for periods from the single channel search are depicted as open squares with $3 \sigma$ error bars

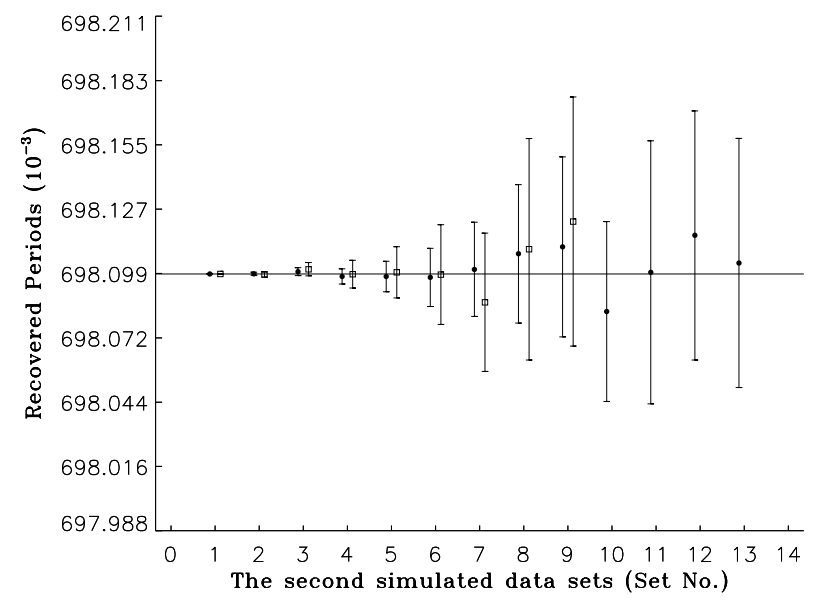

Fig. 9. Multi and single channel search results for the second group of simulations. See detailed explanations in Fig. 8

\section{Discussion and conclusions}

The three stage weighted MPA has been developed especially for simultaneous multichannel photometric observations. The new method has been tested successfully using two groups of artificially generated data with varying noise levels. The light curve model for the first group was constructed arbitrarily. The model for the second group was built using real-life multichannel photometric measurements as a template. Here are the main conclusions which can be drawn from our experiments:

1. The MPA allows one to take into account all available data in a uniform and most informative way. It works correctly with different light curves and different noise levels in different channels. It incorporates easily data sets with missing values in some particular channels; 
2. The theoretically predicted improvement in detection capabilities and in estimation precision of the multichannel method was proved using simple simulations. The use of the new method for real data sets will be described elsewhere;

3. It is possible to construct multichannel variants for all stages of the periodicity search. The grid search and the final period refinement can be done in the framework of standard least squares estimation. The only difference is the use of combined sums of squares. All the new methods described above can easily be implemented by introducing minor extensions to available software;

4. We point out that the interpretation of the PDM spectra is somewhat more complicated than that of the classical power spectra. Thus, we derived the general formula for spurious periods (Eq. (19)) to help the identification of the correct periods among the ghosts.

In this paper only a single period case is treated. Multiperiodic light curves and especially coupled periods will complicate the analysis significantly. We can expect that multichannel methods will still give some edge over standard single channel methods.

The proposed method can also be used for searching hidden periodicities in the variations of spectral line profiles (if we use all wavelength pixels of the observed spectra as different photometric channels). It will detect periods even when equivalent widths remain stable and only the spectral profiles change. This kind of time series spectroscopy can reveal even low level periodic disturbances (say, from invisible companions etc.).
Acknowledgements. The work of Jaan Pelt was supported by the Estonian Science Foundation grant 2628. The authors thank Dr. Rudolf Dümmler and Dr. Andrei Berdyugin for checking the text, and the anonymous referee for his valuable comments.

\section{References}

Bevington P.R., 1969, Data Reduction and Error Analysis for the Physical Sciences. New York: McGraw-Hill, Inc.

Brent R.P., 1973, Algorithms for Minimization without Derivatives, Englewood Cliffs, NJ: Prentice-Hall, Chapter 5 Deeming T.J., 1975, Ap\&SS 36, 137

Koen C., 1999, MNRAS 309, 769

Jetsu L., Pelt J., 1999, A\&AS 139, 629

Pelt J., 1980, Frequency Analysis of Astronomical Time Series, Valgus Publ. Tallinn, monograph, in Russian

Pelt J., 1983, in Statistical Methods in Astronomy, Jaschek et al. (eds.), ESA SP-201, 37

Pelt J., 1993, in 5th ESO/ST-ECF Data Analysis Workshop, Grosbøl P.J., Ruijssher R.C.E. (eds.), ESO Conference and Workshop Proceedings No. 47, p. 179

Pelt J., 1997, in Applications of Time Series Analysis in Astronomy and Meteorology, Subba Rao T., Priestly M.B., Lessi O. (eds.). Chapman \& Hall, p. 249

Press W.H., Flannery B.P., Teukolsky S.A., Vetterling W.T., Flannery B.P., 1994, Numerical Recipes: The Art of Scientific Computing (in Fortran). New York: Cambridge University Press, 2nd ed., repr. with corr.

Shevchenko V.S., 1980, Astr. Zh. 57, 1162 (SvA 24, 670)

Tanner R.W., 1948, Comm. David Dunlap Obs., No. 16

You J., 1999, Time Series Analysis of Brightness Variations of Stars, Licentiate thesis, University of Oulu 